\title{
Prevalence of thinness, overweight and obesity among Tibetan adolescents aged 12-17 years
}

\author{
Yunfei Zhang ${ }^{1}$, Jiahong Sun ${ }^{1}$, Min Zhao ${ }^{2}$, Costan G Magnussen ${ }^{3,4,5}$ and Bo $\mathrm{Xi}^{1, *}$ \\ 'Department of Epidemiology, School of Public Health, Cheeloo College of Medicine, Shandong University, \\ 44 Wenhuaxi Road, Jinan 250012, Shandong, People's Republic of China: ${ }^{2}$ Department of Nutrition and Food \\ Hygiene, School of Public Health, Cheeloo College of Medicine, Shandong University, Jinan, Shandong, People's \\ Republic of China: ${ }^{3}$ Menzies Institute for Medical Research, University of Tasmania, Hobart, Tasmania, Australia: \\ ${ }^{4}$ Research Centre of Applied and Preventive Cardiovascular Medicine, University of Turku, Turku, Finland: ${ }^{5}$ Centre for \\ Population Health Research, University of Turku and Turku University Hospital, Turku, Finland
}

Submitted 25 April 2020: Final revision received 21 December 2020: Accepted 15 January 2021: First published online 21 January 2021

\begin{abstract}
Objective: To estimate the prevalence of thinness, overweight and obesity among Tibetan adolescents aged 12-17 years.

Design: Cross-sectional survey.

Setting: Shigatse City of Tibet municipality, with an average altitude of more than $4000 \mathrm{~m}$.

Participants: Study participants included 2642 adolescents aged 12-17years selected from six schools using a convenient cluster sampling method.

Results: The prevalence of thinness/overweight/obesity among Tibetan adolescents was $9.4 \% / 5.4 \% / 1.4 \%$ (China definition), $14.7 \% / 4.4 \% / 0.7 \%$ (International Obesity Task Force (IOTF) definition), and 2.8\%/5.7\%/0.9\% (WHO definition). The prevalence of thinness and overweight was significantly different between both sexes based on each of three BMI classification criteria $(P<0 \cdot 001)$. There was no significant difference in the prevalence of obesity between both sexes according to each of three BMI criteria. There was no clear trend in the prevalence of thinness across ages according to the China or IOTF definition (both $P>0.05$ ), whereas an upward trend was observed for thinness in boys according to the IOTF definition $\left(P_{\text {for trend }}<0.05\right)$. In contrast, the prevalence of thinness tended to decrease with increasing age in girls according to the IOTF definition and in total sample according to the WHO definition $\left(P_{\text {for trend }}<0.05\right)$.

Conclusions: Among Tibetan adolescents, the prevalence of overweight and obesity is relatively low, while the prevalence of thinness is high, especially in boys. These data suggest urgent attention is needed to control adolescent thinness in Tibet.
\end{abstract}



At present, BMI is widely used as an indicator to evaluate the nutritional status of children and adolescents in the world ${ }^{(1,2)}$. Being overweight or obese during childhood or adolescence is associated with short-term and long-term risk of adverse health outcomes such as hypertension, dyslipidaemia ${ }^{(3)}$, target organ damage ${ }^{(4)}$ and $\mathrm{CVD}^{(5)}$. Recently, the prevalence of overweight and obesity among children and adolescents has been increasing around the world, including in China ${ }^{(1,6-8)}$. For instance, between 1997 and 2011 in China, the prevalence of overweight (including obesity) among children and adolescents aged 7-17years increased from 6.5 to $15.5 \%$ in boys and from 4.6 to $10.4 \%$ in girls ${ }^{(6)}$, and between
2011 and 2015, the prevalence of overweight (including obesity) remained stable in children aged $7-11$ years but continued to increase in adolescents aged $12-18$ years ${ }^{(9)}$. Despite these increasing trends at the upper end of the BMI distribution, the prevalence of stunting or thinness remains prevalent in children living in developing countries ${ }^{(10)}$. In rural areas or some remote provinces of China, there was a relatively high prevalence of stunting and thinness. For example, in Guizhou province in 2014, the prevalence of stunting among children and adolescents aged $7-18$ years was $8.9 \%$ in boys and $9.0 \%$ in girls, and the prevalence of thinness was $5.6 \%$ in boys and $3.9 \%$ in $\operatorname{girls}^{(7)}$. 
The Tibet Autonomous Region of China is located at high altitude in the Qing-Tibetan highland. The hypoxic environments, socio-economic conditions and lifestyle factors in high altitude areas may have significant impacts on children's growth and development ${ }^{(11)}$. The majority of Tibetan children and adolescents live at high altitudes which may lead to the unhealthy nutritional status among them ${ }^{(12)}$. Several studies have shown a high prevalence of stunting, thinness and underweight among Tibetan children aged $<10$ years $^{(12-14)}$, indicating that child malnutrition is positively related to high altitude ${ }^{(14)}$. However, up to date, no study has focused on the nutritional status of Tibetan adolescents aged 12-17 years living at high altitude. We have a hypothesis that Tibetan adolescents living at high altitude areas may be more likely to have thinness.

Using data from one cross-sectional survey conducted between August and September in 2018 in Shigatse City, Tibet municipality, we aimed to assess the prevalence of thinness, overweight and obesity among Tibetan adolescents aged $12-17$ years.

\section{Methods}

\section{Study population}

Data were from a cross-sectional survey conducted from August to September in 2018 in Shigatse City (with an average altitude of more than $4000 \mathrm{~m}^{(15)}$ ), Tibet municipality. Using a convenient cluster sampling method, we selected three junior high schools in Yadong County, Gamba County and Sangzhutun District of Shigatse City, and three senior high schools in Sangzhutun District of Shigatse City (as senior high schools are usually located in urban regions of Shigatse City). The six high schools were conveniently selected due to their good compliance and cooperation. All students in selected schools were invited to participate in the survey. In this survey, 3250 participants aged 1024 years with complete physical and lifestyle measurements were initially recruited. The ethnic classification of a participant was determined according to the identity certificate information provided by the school. After the completion of data collection, we excluded those who were not Tibetan, younger than 12 years or older than 17 years and those with missing data on physical or lifestyle measurements, and thus a total of 2642 Tibetan adolescents aged 12-17 years with complete data on sex, age, height, weight and lifestyle variables were finally included. Signed informed consent was obtained from each participant and his/her guardians/parents.

\section{Measurements}

Height and weight were measured using the HGM 300 ultrasonic height and weight scale in light clothes without shoes to the nearest $0.1 \mathrm{~cm}$ and $0.1 \mathrm{~kg}$, respectively. BMI was calculated as weight in kilograms divided by the square of height in meters. Height and weight were measured twice, respectively, and the average value was used in the analyses.

Nutritional status was defined according to each of three BMI classifications: the China definition ${ }^{(16,17)}$, the International Obesity Task Force (IOTF) definition ${ }^{(18)}$ and the WHO definition ${ }^{(19)}$. Based on the China definition ${ }^{(16,17)}$, overweight and obesity were defined according to the percentile cut-offs by sex and age in Chinese school-age children and adolescents, and thinness was defined according to percentile thresholds for thinness of school-age children and adolescents. Based on the IOTF definition, thinness, overweight and obesity BMI percentile cut-offs were defined as the linkage of international growth curve data for boys and girls with adult BMI cut-offs of $18.5 \mathrm{~kg} / \mathrm{m}^{2}$ (thin), BMI $25 \mathrm{~kg} / \mathrm{m}^{2}$ (overweight) and BMI $30 \mathrm{~kg} / \mathrm{m}^{2}$ (obese) at the age of 18 years ${ }^{(18)}$. Based on the WHO definition, thinness, overweight and obesity were defined as BMI for age and sex $<-2$ SD, $\geq 1$ SD and $<2$ SD, and $\geq 2$ SD above the WHO Growth Reference median value, respectively $^{(19)}$. The IOTF definition and WHO definition are two widely used international definitions to assess the nutritional status in children and adolescents. The China definition is widely used in Chinese children and adolescents. In the current study, three different definitions were used to assess the prevalence of thinness, overweight and obesity among Tibetan adolescents aged 12-17 years, which can comprehensively reflect the nutritional status among Tibetan adolescents, and can be convenient for prevalence comparison across different regions and countries.

Socio-demographic and lifestyle factors were collected using a self-reported questionnaire, which was distributed by trained investigators to ensure the collection of reliable data. Socio-demographic factors included sex, age and race. Lifestyle factors included intakes of fruit, vegetable and meat, water drinking, physical activity, screen time and sleep duration.

\section{Statistical analysis}

Continuous variables including age, height, weight and BMI were expressed as mean and SD, and sex difference was examined using the $t$-test. Categorical variables including nutritional status, intakes of fruit, vegetable or meat $(<1$ $v . \geq 1$ time/d), water drinking ( $\leq 1 v .>1$ time/d), physical activity $(<1 v . \geq 1$ time/d), screen time $(\leq 2 v .>2 \mathrm{~h} / \mathrm{d})$ and sleep duration ( $<8 v$. $\geq 8 \mathrm{~h} / \mathrm{d}$ ) were expressed as proportions, and sex difference was examined using the $\chi^{2}$ test. General linear regression models were used to examine the trend of BMI with increase of age, in which BMI was the dependent variable and age was the independent variable. Logistic regression models were used to examine the trends in prevalence of thinness, overweight or obesity with increase of age, in which thinness, overweight and obesity, respectively, were the dependent variables, and age was the independent variable, and the cluster effect (i.e., class) was considered in the model. Multivariate 
logistic regression models were used to examine the association between lifestyle factors and nutritional status. All statistical analyses were performed using SAS software (version 9.4). A two-sided $P<0.05$ was considered statistically significant.

\section{Results}

A total of 2642 Tibetan adolescents ( $44.4 \%$ boys) were included in our study. The mean age of the surveyed participants was 15.3 (SD 1.7) years. Boys were taller than girls (158.3 v. $153.8 \mathrm{~cm} ; P<0.001)$. However, girls had greater mean values of BMI than boys $\left(19.9 v .18 .6 \mathrm{~kg} / \mathrm{m}^{2}\right.$; $P<0.001$ ) (Table 1 ). Girls were more likely to consume vegetable one or more times per $\mathrm{d}$, to sleep $<8 \mathrm{~h} / \mathrm{d}$ and to spend screen time more than $2 \mathrm{~h} / \mathrm{d}$. Boys were more likely to consume meat one or more times per $\mathrm{d}$ and to perform physical activity one or more times per $\mathrm{d}$. However, there was no significant difference in weight, fruit intake and water drinking between both sexes (online supplementary material, Supplemental Table S1).

As shown in Table 2, BMI showed an upward trend with increase of age among both sexes (both $P_{\text {for trend }}<0.001$ ). Based on the China, IOTF and WHO definitions, the prevalence of overweight/obesity among Tibetan adolescents was $5.4 \% / 1.4 \%, 4.4 \% / 0.7 \%$ and $5.7 \% / 0.9 \%$, respectively, while the prevalence of thinness was $9.4,14.7$ and $2.8 \%$, respectively (Fig. 1). Table 3 presents the prevalence of thinness, overweight and obesity among Tibetan adolescents according to sex and three BMI classifications. The prevalence of thinness and overweight was significantly different between sexes according to each of three BMI classifications $(P<0 \cdot 001)$. There was no significant difference in the prevalence of obesity between both sexes according to each of three BMI classifications.

As shown in online supplementary material, Supplemental Table S2, there was no significant trend in thinness with increase of age according to the China and IOTF definitions, but the prevalence of thinness tended to increase with increase of age in boys according to the IOTF definition $\left(P_{\text {for trend }}<0.05\right)$. In contrast, the prevalence of thinness tended to decrease with increase of age in girls according to the IOTF definition and in total sample according to the WHO definition $\left(P_{\text {for trend }}<0.05\right)$. There were no significant trends in overweight or obesity according to the China, IOTF and WHO definitions.

Online supplementary material, Supplemental Table S3 presents potential lifestyle factors associated with thinness, overweight and obesity among Tibetan adolescents. Short sleep duration was associated with reduced risk of thinness. Tibetan adolescents who consumed fruits less than one time per $\mathrm{d}$ had an increased risk of overweight. Tibetan adolescents who spent screen time more than $2 \mathrm{~h} / \mathrm{d}$ had an increased risk of overweight.

\section{Discussion}

Our findings showed a relatively low prevalence of overweight and obesity and a high prevalence of thinness among Tibetan adolescents living at high altitude. The prevalence of thinness differed substantially depending on the classification used to assign nutritional status, while only minor differences were found for the prevalence of overweight and obesity. The difference in prevalence of

Table 1 Characteristics of the study population*

\begin{tabular}{|c|c|c|c|c|c|c|c|}
\hline \multirow[b]{2}{*}{ Characteristics } & \multicolumn{2}{|c|}{ Total (n 2642) } & \multicolumn{2}{|c|}{ Boys (n 1174) } & \multicolumn{2}{|c|}{ Girls (n 1468) } & \multirow[b]{2}{*}{$P$} \\
\hline & Mean & SD & Mean & SD & Mean & SD & \\
\hline Age (years) & $15 \cdot 3$ & $1 \cdot 7$ & $15 \cdot 1$ & 1.6 & $15 \cdot 4$ & $1 \cdot 7$ & $<0.001$ \\
\hline Height (cm) & $155 \cdot 8$ & $8 \cdot 6$ & $158 \cdot 3$ & $10 \cdot 5$ & $153 \cdot 8$ & $6 \cdot 0$ & $<0.001$ \\
\hline Weight (kg) & $47 \cdot 1$ & 8.9 & $47 \cdot 0$ & 9.9 & $47 \cdot 2$ & $8 \cdot 0$ & 0.609 \\
\hline BMI $\left(\mathrm{kg} / \mathrm{m}^{2}\right)$ & $19 \cdot 3$ & $2 \cdot 6$ & $18 \cdot 6$ & $2 \cdot 3$ & $19 \cdot 9$ & $2 \cdot 6$ & $<0.001$ \\
\hline
\end{tabular}

${ }^{*}$ Continuous variables are presented as mean and SD, and sex difference was examined using the $t$-test.

Table 2 BMl $\left(\mathrm{kg} / \mathrm{m}^{2}\right)$ of Tibetan adolescents aged $12-17$ years by age and gender*

\begin{tabular}{|c|c|c|c|c|c|c|c|c|c|c|c|c|c|}
\hline \multirow[b]{3}{*}{ Sex } & \multicolumn{13}{|c|}{ Age (years) } \\
\hline & \multicolumn{2}{|c|}{$12(n$ 303) } & \multicolumn{2}{|c|}{$13(n 435)$} & \multicolumn{2}{|c|}{$14(n 430)$} & \multicolumn{2}{|c|}{$15(n 401)$} & \multicolumn{2}{|c|}{$16(n 551)$} & \multicolumn{2}{|c|}{$17(n 522)$} & \multirow[b]{2}{*}{$P_{\text {for trend }} \uparrow$} \\
\hline & Mean & $\mathrm{SD}$ & Mean & $\mathrm{SD}$ & Mean & $\mathrm{SD}$ & Mean & SD & Mean & $\mathrm{SD}$ & Mean & SD & \\
\hline Total & $17 \cdot 9$ & $2 \cdot 4$ & $18 \cdot 0$ & $2 \cdot 2$ & $19 \cdot 0$ & 2.4 & $19 \cdot 6$ & $2 \cdot 4$ & $20 \cdot 1$ & 2.6 & $20 \cdot 4$ & $2 \cdot 3$ & $<0.001$ \\
\hline Boys & $17 \cdot 8$ & 2.5 & $17 \cdot 5$ & $2 \cdot 2$ & $18 \cdot 2$ & 1.9 & $18 \cdot 8$ & $2 \cdot 0$ & $19 \cdot 2$ & $2 \cdot 2$ & $19 \cdot 6$ & $2 \cdot 2$ & $<0.001$ \\
\hline Girls & $17 \cdot 9$ & $2 \cdot 3$ & $18 \cdot 4$ & $2 \cdot 2$ & $19 \cdot 7$ & $2 \cdot 6$ & $20 \cdot 4$ & $2 \cdot 4$ & $20 \cdot 7$ & 2.7 & $20 \cdot 8$ & $2 \cdot 2$ & $<0.001$ \\
\hline
\end{tabular}

*Data are presented as mean and SD.

$\dagger P_{\text {for trend }}($ age) was tested using linear regression analysis. 


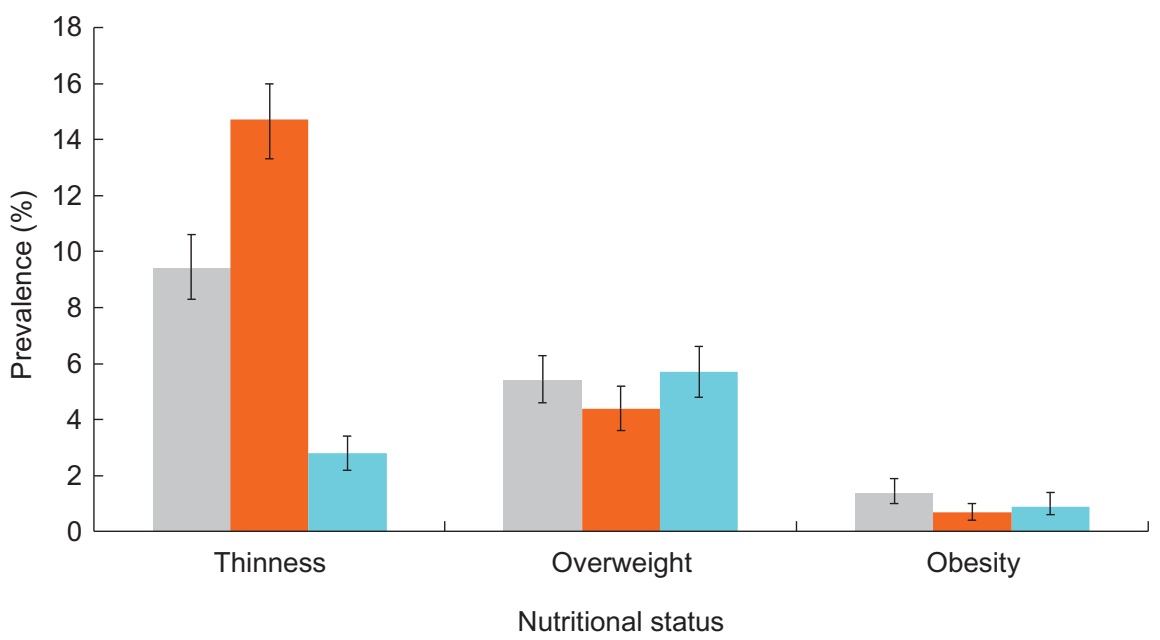

Fig. 1 (colour online) Prevalence (\%) of thinness, overweight and obesity among Tibetan adolescents by different BMI classifications. , China definition; $\div$, IOTF definition; $\div$, WHO definition

Table 3 Prevalence of thinness, overweight and obesity among Tibetan adolescents by gender and different BMI classifications

\begin{tabular}{|c|c|c|c|c|c|c|c|}
\hline \multirow[b]{2}{*}{ Sex } & \multirow[b]{2}{*}{ Nutritional status } & \multicolumn{6}{|c|}{ BMI definitions (\%) } \\
\hline & & China definition & $95 \% \mathrm{Cl}$ & IOTF definition & $95 \% \mathrm{Cl}$ & WHO definition & $95 \% \mathrm{Cl}$ \\
\hline \multirow[t]{3}{*}{ Boys } & Thinness & $16 \cdot 5$ & $14 \cdot 4,18 \cdot 7$ & $18 \cdot 6$ & $16 \cdot 3,20 \cdot 8$ & 4.6 & $3 \cdot 4,5 \cdot 8$ \\
\hline & Overweight & $3 \cdot 2$ & $2 \cdot 2,4 \cdot 3$ & 2.9 & $1.9,3.9$ & $3 \cdot 3$ & $2 \cdot 3,4 \cdot 4$ \\
\hline & Obesity & $1 \cdot 3$ & $0.6,1.9$ & 0.8 & $0.3,1 \cdot 3$ & $1 \cdot 3$ & $0.6,1.9$ \\
\hline \multirow[t]{3}{*}{ Girls } & Thinness & $3 \cdot 8$ & $2 \cdot 8,4.7$ & $11 \cdot 6$ & $9 \cdot 9,13 \cdot 2$ & 1.4 & $0 \cdot 8,2 \cdot 0$ \\
\hline & Overweight & $7 \cdot 2$ & $5 \cdot 8,8.5$ & 5.7 & $4 \cdot 5,6 \cdot 8$ & $7 \cdot 6$ & $6 \cdot 2,8 \cdot 9$ \\
\hline & Obesity & 1.5 & $0.9,2.1$ & 0.6 & $0.2,1.0$ & 0.6 & $0.2,1.0$ \\
\hline $\begin{array}{l}P \text { for thinness difference } \\
\text { between genders }\end{array}$ & & $<0.001$ & & $<0.001$ & & $<0.001$ & \\
\hline $\begin{array}{l}P \text { for overweight difference } \\
\text { between genders }\end{array}$ & & $<0.001$ & & $<0.001$ & & $<0.001$ & \\
\hline $\begin{array}{l}P \text { for obesity difference } \\
\text { between genders }\end{array}$ & & 0.631 & & 0.634 & & 0.074 & \\
\hline
\end{tabular}

IOTF, International Obesity Task Force.

thinness might be due to that the IOTF definition is based on data collected from six countries ${ }^{(18)}$, the WHO definition is based on data collected from the USA alone ${ }^{(19)}$ and the China definition is based on data collected from China. There are differences in living environments and lifestyle behaviours between the participants used for the development of three definitions, and thus they result in different BMI percentile cut-offs for three different definitions. But the current use of three definitions will make our results comparable with participants from China and outside China.

The prevalence of overweight and obesity in our study is lower than that reported for Chinese children and adolescents between 2011 and 2015, based on the IOTF and WHO definitions ${ }^{(9)}$, and is also lower than what has been reported in developed countries based on the IOTF definition $^{(8)}$. One cross-sectional study in China indicated that the prevalence of excess body weight (including overweight and obesity) in Tibetan adolescents aged $7-18$ years was $12.0 \%$ (using the China definition) ${ }^{(2)}$, which is higher than our findings (the prevalence was $6.9 \%$ using the same definition). However, findings from one previous study were consistent with ours with the prevalence of overweight and obesity in children aged 9-10 years in Lhasa was 3.5 and $0.5 \%$ in girls and 4.8 and $1.5 \%$ in boys, based on the WHO definition ${ }^{(13)}$.

One study showed that the prevalence of moderate and severe thinness in children and adolescents aged 5-19years worldwide in 2016 was $8.4 \%$ in girls and $12.4 \%$ in boys based on the WHO definition ${ }^{(1)}$, and a large national successive cross-sectional survey in China showed that the prevalence of stunting decreased from $16.4 \%$ in 1985 to $2 \cdot 3 \%$ in 2014 , and the prevalence of thinness decreased from $8.4 \%$ in 1985 to $4.0 \%$ in 2014 based on the WHO definition ${ }^{(7)}$. Compared with the above results, the prevalence of thinness in our study is lower than that in children and adolescents worldwide but higher than the average level in China. A study in 2018 reported that the prevalence of thinness in Tibetan adolescents aged $7-18$ years was $14.9 \%$ in boys and $9.2 \%$ in girls based 
on the China definition ${ }^{(2)}$, which is similar to our findings for boys but higher than girls in our study. Another recent review of 398 Tibetan children aged 9-10 years showed that the prevalence of underweight was $36.7 \%$ in girls and $31.1 \%$ in boys based on the WHO definition, which is much higher than what we found ${ }^{(13)}$. The discrepancy in these findings might be due to differences in age range, sample size and sampling methods. To our knowledge, no study has assessed the nutritional status based on different BMI definitions in Tibetan adolescents aged $12-17$ years. Although the proportions differ between studies mentioned above, these data consistently show that there is still a high prevalence of thinness in Tibetan children and adolescents. One reason is the adverse influence of poor socioeconomic conditions in Tibet ${ }^{(12,20)}$, while others have emphasised that a high altitude might lead to delayed growth in children ${ }^{(14,21)}$. In addition, the economic environment of localities at high altitude is often poor, which can lead to a high prevalence of thinness in children and adolescents $^{(11)}$

Irrespective of the different BMI classifications applied in our study, the prevalence of thinness was higher in boys than girls, while the prevalence of overweight was higher in girls than boys. Findings from previous studies are similar to ours ${ }^{(2,22)}$, while some others have shown an opposite trend $^{(13)}$. The following reasons might explain the sex difference in nutritional status. Compared with girls, boys usually engage in more physical activities (e.g., playing basketball and footballs) ${ }^{(23)}$. The gender difference in nutritional status might also be explained by an earlier onset of sexual maturation among girls, as fat accumulation is more likely to occur in girls after 12 years of age ${ }^{(2)}$, and body fat percentage is indeed positively correlated with $\mathrm{BMI}^{(24)}$.

Although we found no significant age trend in the prevalence of thinness according to the China and IOTF definitions, the prevalence of thinness tended to decrease with increase of age according to the WHO definition. There were no obvious trends with increase of age for the prevalence of overweight and obesity according to the China, IOTF and WHO definitions. A study reported that the prevalence of thinness among Chinese children with ethnic minority aged 7-9 years is highest, according to the China definition, followed by adolescents with ethnic minority aged 16-18 and 13-15 years ${ }^{(2)}$. Another study in fifty-eight low- and middle-income countries showed that the prevalence of overweight and obesity among young adolescents aged $12-13$ years is higher than those aged $14-15$ years according to the IOTF and WHO definitions, but the prevalence of thinness among those aged 12-13 years is lower than those aged $14-15$ years ${ }^{(25)}$. The inconsistency of these findings might be explained by differences in race, sample sizes and sampling methods.

We found that several lifestyle factors were associated with thinness, overweight and obesity among Tibetan adolescents. Tibetan adolescents who spent screen time more than $2 \mathrm{~h} / \mathrm{d}$ had an increased risk of overweight, which is similar to the findings from previous studies ${ }^{(26,27)}$. In addition, short sleep duration was associated with reduced risk of thinness in our study, and the same result was found in one adult study ${ }^{(28)}$. We also found that Tibetan adolescents who consumed fruits less than once per day had an increased risk of overweight. Similar to our finding, a systematic review showed that there was a significantly inverse association between intakes of fruits and vegetables and abdominal obesity among adolescents ${ }^{(29)}$.

The main strength of the current study includes the use of three BMI classifications (China, IOTF and WHO definitions) to estimate the prevalence of thinness, overweight and obesity in Tibetan adolescents, which allows a direct comparison of our findings with those from others. However, the current study has some limitations. Firstly, thinness can only indicate an unhealthy nutritional status ${ }^{(30)}$. Further studies with more detailed information of malnutrition status are needed. Secondly, BMI is a crude indicator of nutritional status, and other indicators such as body fat percentage and biochemical indicators should be considered in future studies. Thirdly, the current study used a convenient cluster sampling method, and the representativeness of the data might have been affected. Fourthly, several variables including sleep time, screen time, intakes of fruit, vegetable and meat, water drinking and exercise were self-reported, which might have affected the observed association.

In conclusion, we found the prevalence of overweight and obesity is low among Tibetan adolescents aged 1217 years, but the prevalence of thinness is generally high, especially among boys. Our findings reinforce evidence suggesting that timely monitoring and improvement of the nutritional status of thinness among Tibetan adolescents are necessary, although the co-occurrence of overweight and obesity also should be paid some attention.

\section{Acknowledgements}

Acknowledgements: The authors thank all students and their parents who participated in this survey. Financial support: This work was supported by Cheeloo Medical School, Shandong University. Conflict of interest: The authors declare that they have no competing interests. Authorship: B.X. designed and supervised the study. Y.Z. analysed the data and drafted the manuscript. B.X., C.G.M., M.Z. and J.S. made contributions to interpretation of data and critically revised the manuscript. All authors read and approved the submission of the final manuscript. Ethics of human subject participation: The current study was conducted according to the guidelines laid down in the Declaration of Helsinki and all procedures involving human subjects were approved by the Ethics Committee of Shandong University. Written informed consent was obtained from all subjects. 


\section{Supplementary material}

For supplementary material accompanying this paper visit https://doi.org/10.1017/S1368980021000215

\section{References}

1. Abarca-Gómez L, Abdeen ZA, Hamid ZA et al. (2017) Worldwide trends in body-mass index, underweight, overweight, and obesity from 1975 to 2016: a pooled analysis of 2416 population-based measurement studies in 1289 million children, adolescents, and adults. Lancet $\mathbf{3 9 0}$, 2627-2642.

2. Dong Y, Zou Z, Yang Z et al. (2018) Prevalence of excess body weight and underweight among 26 Chinese ethnic minority children and adolescents in 2014: a cross-sectional observational study. BMC Public Health 18, 562.

3. Friedemann C, Heneghan C, Mahtani K et al. (2012) Cardiovascular disease risk in healthy children and its association with body mass index: systematic review and metaanalysis. BMJ 345, e4759.

4. Cote AT, Harris KC, Panagiotopoulos C et al. (2013) Childhood obesity and cardiovascular dysfunction. $J$ Am Coll Cardiol 62, 1309-1319.

5. Llewellyn A, Simmonds M, Owen CG et al. (2016) Childhood obesity as a predictor of morbidity in adulthood: a systematic review and meta-analysis. Obes Rev 17, 56-67.

6. Wang H, Xue H, Du S et al. (2017) Time trends and factors in body mass index and obesity among children in China: 1997-2011. Int J Obes 41, 964-970.

7. Song Y, Agardh A, Ma J et al. (2019) National trends in stunting, thinness and overweight among Chinese school-aged children, 1985-2014. Int J Obes 43, 402-411.

8. Ng M, Fleming T, Robinson M et al. (2014) Global, regional, and national prevalence of overweight and obesity in children and adults during 1980-2013: a systematic analysis for the Global Burden of Disease Study 2013. Lancet $\mathbf{3 8 4}$ 766-781.

9. Zhang J, Wang H, Wang Z et al. (2018) Prevalence and stabilizing trends in overweight and obesity among children and adolescents in China, 2011-2015. BMC Public Health 18, 571.

10. de Onis M, Onyango AW, Borghi E et al. (2006) Comparison of the World Health Organization (WHO) child growth standards and the national center for health statistics/WHO international growth reference: implications for child health programmes. Public Health Nutr 9, 942-947.

11. Niermeyer S, Andrade Mollinedo P \& Huicho L (2009) Child health and living at high altitude. Arch Dis Child 94, 806-811.

12. Harris NS, Crawford PB, Yangzom Y et al. (2001) Nutritional and health status of Tibetan children living at high altitudes. $N$ Engl J Med 344, 341-347.

13. Bianba B, Yangzong Y, Gonggalanzi G et al. (2015) Anthropometric measures of 9- to 10-year-old native Tibetan children living at 3700 and $4300 \mathrm{~m}$ above sea level and Han Chinese living at $3700 \mathrm{~m}$. Medicine 94, e1516.
14. Dang S, Yan H, Yamamoto S et al. (2004) Poor nutritional status of younger Tibetan children living at high altitudes. EurJ Clin Nutr 58, 938-946.

15. Liu R (1988) China Population, Section of Tibet. Beijing: Publishing House of Finance and Economy.

16. National Health and Family Planning Commission of China (NHFPC) (2018) Screening standard for overweight and obesity of school-age children and adolescents. http:// www.nhfpc.gov.cn/wjw/pqt/201803/a7962d1ac01647b9837 110bfd2d69b26.shtml (accessed October 2020).

17. National Health and Family Planning Commission of China (NHFPC) (2014) Screening standard for malnutrition of school-age children and adolescents. http://www.moh.gov. cn/zwgkzt/pqt/201407/38b15c0a1ed444e8908e12752decaffa (accessed October 2020).

18. Cole TJ \& Lobstein T (2012) Extended international (IOTF) body mass index cut-offs for thinness, overweight and obesity. Pediatr Obes 7, 284-294.

19. de Onis M, Onyango AW, Borghi E et al. (2007) Development of a WHO growth reference for school-aged children and adolescents. Bull World Health Organ 85, 660-667.

20. Pawson IG, Huicho L, Muro M et al. (2010) Growth of children in two economically diverse Peruvian high-altitude communities. Am J Hum Biol 13, 323-340.

21. Greksa LP (2006) Growth and development of Andean high altitude residents. High Alt Med Biol 7, 116-124.

22. Gaskin PS \& Walker SP (2003) Obesity in a cohort of black Jamaican children as estimated by BMI and other indices of adiposity. Eur J Clin Nutr 57, 420-426.

23. Li H, Li X, Wang Y et al. (2013) Objective assessment of school-time physical activity of a grade 1-2 junior school students in Beijing. J Hygien Res 42, 589-595.

24. Wang J, Wang H, Liu J et al. (2013) The association between body mass index, waist circumference with body fat percent, and abdominal fat rate in overweight and obese pupils. Chin J Prev Med 47, 603-607.

25. Yang L, Bovet P, Ma C et al. (2018) Prevalence of underweight and overweight among young adolescents aged 12-15 years in 58 low-income and middle-income countries. Pediatr Obes 14, e12468.

26. Braithwaite I, Stewart AW, Hancox RJ et al. (2013) The worldwide association between television viewing and obesity in children and adolescents: cross sectional study. PLoS One 8, e74263.

27. Marshall SJ, Biddle SJ, Gorely T et al. (2004) Relationships between media use, body fatness and physical activity in children and youth: a meta-analysis. Int J Obes Relat Metab Disord 28, 1238-1246.

28. Ryu SY, Kim KS, Han MA (2011) Factors associated with sleep duration in Korean adults: results of a 2008 community health survey in Gwangju metropolitan city, Korea. J Korean Med Sci 26, 1124-1131.

29. Collese TS, Nascimento-Ferreira MV, de Moraes ACF et al. (2017) Role of fruits and vegetables in adolescent cardiovascular health: a systematic review. Nutr Rev 75, 339-349.

30. Li Y, Hu X, Zhao J et al. (2009) Application of the WHO growth reference (2007) to assess the nutritional status of children in China. Biomed Environ Sci 22, 130-135. 